\title{
Any two-dimensional Normed space is a generalized Day-James space
}

Javier Alonso

Correspondence: jalonso@unex.es Department of Mathematics, University of Extremadura, 06006 Badajoz, Spain

\section{Abstract}

It is proved that any two-dimensional normed space is isometrically isomorphic to a generalized Day-James space $\ell_{\psi}-\ell_{\phi}$ introduced by W. Nilsrakoo and S. Saejung.

Keywords: Normed space, Day-James space, Birkhoff orthogonality

\section{Mathematics Subject Classification 46B20}

The Day-James space $\ell_{p}-\ell_{q}$ is defined for $1 \leq p, q \leq \infty$ as the space $\mathbb{R}^{2}$ endowed with the norm

$$
\|x\|_{p, q}=\left\{\begin{array}{l}
\|x\|_{p} \text { if } x_{1} x_{2} \geq 0 \\
\|x\|_{q} \text { if } x_{1} x_{2} \leq 0,
\end{array}\right.
$$

where $x=\left(x_{1}, x_{2}\right)$. James [1] considered the space $\ell_{p}-\ell_{q}$ with $1 / p+1 / q=1$ as an example of a two-dimensional normed space where Birkhoff orthogonality is symmetric. Recall that if $x$ and $y$ are vectors in a normed space then $x$ is said to be Birkhoff orthogonal to $y$, $\left(x \perp_{B} y\right)$, if $\|x+\lambda y\| \geq\|x\|$ for every scalar $\lambda$ [2]. Birkhoff orthogonality coincides with usual orthogonality in inner product spaces. In arbitrary normed spaces Birkhoff orthogonality is in general not symmetric (e.g., in $\mathbb{R}^{2}$ with $\|\cdot\|_{\infty}$ ), and it is symmetric in a normed space of three or more dimension if and only if the norm is induced by an inner product. This last significant property was obtained in gradual stages by Birkhoff [2], James [1,3], and Day [4]. The first reference related to the symmetry of Birkhoff orthogonality in two-dimensional spaces seems to be Radon [5] in 1916. He considered plane convex curves with conjugate diameters (as in ellipses) in order to solve certain variational problems.

The procedure that James used to get two-dimensional normed spaces where Birkhoff orthogonality is symmetric was extended by Day [4] in the following way. Let $\left(X,\|\cdot\|_{X}\right)$ be a two-dimensional normed space and let $u, v \in X$ be such that $\|u\|_{X}=\|v\|_{X}=1, u \perp_{B} v$, and $v \perp_{B} u$ (see Lemma below). Then, taking a coordinate system where $u=(1,0)$ and $v=$ $(0,1)$ and defining

$$
\left\|\left(x_{1}, x_{2}\right)\right\|_{X, X^{*}}= \begin{cases}\left\|\left(x_{1}, x_{2}\right)\right\|_{X} & \text { if } x_{1} x_{2} \geq 0 \\ \left\|\left(x_{1}, x_{2}\right)\right\|_{X^{*}} & \text { if } x_{1} x_{2} \leq 0\end{cases}
$$

one gets that in the space $\left(X,\|\cdot\|_{X, X} *\right)$ Birkhoff orthogonality is symmetric. Moreover, Day also proved that surprisingly the norm of any two-dimensional space where Birkhoff orthogonality is symmetric can be constructed in the above way.

(C) 2011 Alonso; licensee Springer. This is an Open Access article distributed under the terms of the Creative Commons Attribution License (http://creativecommons.org/licenses/by/2.0), which permits unrestricted use, distribution, and reproduction in any medium, provided the original work is properly cited. 
A norm on $\mathbb{R}^{2}$ is called absolute if $\left\|\left(x_{1}, x_{2}\right)\right\|=\left\|\left(\left|x_{1}\right|,\left|x_{2}\right|\right)\right\|$ for any $\left(x_{1}, x_{2}\right) \in \mathbb{R}^{2}$. Following Nilsrakoo and Saejung [6] let $A N_{2}$ be the family of all absolute and normalized (i.e., $\|(1,0)\|=\|(0,1)\|=1$ ) norms on $\mathbb{R}^{2}$. Examples of norms in AN2 are $\ell_{p}$ norms. Bonsall and Duncan [7] showed that there is a one-to-one correspondence between $A N_{2}$ and the family $\Psi_{2}$ of all continuous and convex functions $\psi:[0,1] \rightarrow \mathbb{R}$ such that $\psi(0)=\psi(1)=1$ and $\max \{1-t, t\} \leq \psi(t) \leq 1(0 \leq t \leq 1)$. The correspondence is given by $\psi(t)=\|(1-t, t)\|$ for $\|\cdot\|$ in $A N_{2}$, and by

$$
\left\|\left(x_{1}, x_{2}\right)\right\|_{\psi}= \begin{cases}\left(\left|x_{1}\right|+\left|x_{2}\right|\right) \psi\left(\frac{\left|x_{2}\right|}{\left|x_{1}\right|+\left|x_{2}\right|}\right) & \text { if }\left(x_{1}, x_{2}\right) \neq(0,0), \\ 0 & \text { if }\left(x_{1}, x_{2}\right)=(0,0) .\end{cases}
$$

for $\psi$ in $\Psi_{2}$.

In [6] the family of norms $\|\cdot\|_{p, q}$ of Day-James spaces $\ell_{p}-\ell_{q}$ is extended to the family $N_{2}$ of norms defined in $\mathbb{R}^{2}$ as

$$
\left\|\left(x_{1}, x_{2}\right)\right\|_{\psi, \varphi}=\left\{\begin{array}{l}
\left\|\left(x_{1}+x_{2}\right)\right\|_{\psi} \text { if } x_{1}, x_{2} \geq 0 \\
\left\|\left(x_{1}+x_{2}\right)\right\|_{\varphi} \text { if } x_{1}, x_{2} \leq 0
\end{array}\right.
$$

for $\psi, \phi L \Psi_{2}$. The space $\mathbb{R}_{2}$ endowed with the above norm is called an $\ell_{\psi}-\ell_{\phi}$ space.

The purpose of this paper is to show that any two-dimensional normed space is isometrically isomorphic to an $\ell_{\psi}-\ell_{\phi}$ space. To this end we shall use the following lemma due to Day [8]. The nice proof we reproduce here is taken from the PhD Thesis of del Río [9], and is based on explicitly developing the idea underlying one of the two proofs given by Day.

Lemma 1 [8]. Let $(X,\|\cdot\|)$ be a two-dimensional normed space. Then, there exist $u, v$ $\in X$ such that $\|u\|=\|v\|=1, u \perp_{B} v$, and $v \perp_{B} u$.

Proof. Let $e, \hat{e} \in X$ be linearly independent, and for $x \in X$ let $\left(x_{1}, x_{2}\right) L \mathbb{R}^{2}$ be the coordinates of $x$ in the basis $\{e, \hat{e}\}$. Let $S=\{x \in X:\|x\|=1\}$, and for $x \in S$ consider the linear functional $f_{x}: y \in X \mapsto f_{x}(y)=x_{2} y_{1}-x_{1} y_{2}$. Then it is immediate to see that $f_{x}$ attains the norm in $y \in S$ (i.e., $\left|x_{2} y_{1}-x_{1} y_{2}\right| \geq\left|x_{2} z_{1}-x_{1} z_{2}\right|$, for all $z_{1} e+z_{2} \hat{e} \in S$ ) if and only if $y \perp_{B} x$. Therefore if $u, v \in S$ are such that $\left|u_{2} v_{1}-u_{1} v_{2}\right|=\max _{(x, y) \in S \times S} \mid x_{2} y_{1}-$ $x_{1} y_{2} \mid$ then $u \perp_{B} v$ and $v \perp_{B} u$.

Theorem 2 For any two-dimensional normed space $\left(X,\|\cdot\|_{X}\right)$ there exist $\psi, \phi L \Psi_{2}$ such that $\left(X,\|\cdot\|_{X}\right)$ is isometrically isomorphic to $\left(\mathbb{R}^{2},\|\cdot\|_{\psi, \phi}\right)$.

Proof. By Lemma 1 we can take $u, v \in X$ such that $\|u\|=\|v\|=1, u \perp_{B} v$, and $v \perp_{B} u$. Then $u$ and $v$ are linearly independent and $\left(X,\|\cdot\|_{X}\right)$ is isometrically isomorphic to $\left(\mathbb{R}^{2}\right.$, $\left.\|\cdot\|_{\mathbb{R} 2}\right)$, where $\left\|\left(x_{1}, x_{2}\right)\right\|_{\mathbb{R}_{2}}:=\left\|x_{1} u+x_{2} v\right\|_{X}$. Defining $\psi(t)=\|(1-t) u+t v\|_{X}, \phi(t)=\|$ $(1-t) u-t v \|_{X},(0 \leq t \leq 1)$, one trivially has that $\psi, \phi \in \Psi_{2}$ and $\left\|\left(x_{1}, x_{2}\right)\right\|_{\mathbb{R} 2}=\|\left(x_{1}, x_{2}\right)$ $\|_{\psi, \phi}$ for all $\left(x_{1}, x_{2}\right) \in \mathbb{R}^{2}$.

Acknowledgements

Research partially supported by MICINN (Spain) and FEDER (UE) grant MTM2008-05460, and by Junta de Extremadura grant GR10060 (partially financed with FEDER).

\section{Competing interests}

The author declares that they have no competing interests. 


\section{References}

1. James, RC: Inner products in normed linear spcaces. Bull Am Math Soc. 53, 559-566 (1947). doi:10.1090/50002-99041947-08831-5

2. Birkhoff, G: Orthogonality in linear metric spaces. Duke Math J. 1, 169-172 (1935). doi:10.1215/50012-7094-35-00115-6

3. James, RC: Orthogonality and linear functionals in normed linear spaces. Trans Am Math Soc. 61, 265-292 (1947). doi:10.1090/S0002-9947-1947-0021241-4

4. Day, MM: Some characterizations of inner product spaces. Trans Am Math Soc. 62, 320-337 (1947). doi:10.1090/S00029947-1947-0022312-9

5. Radon, J: Über eine besondere Art ebener konvexer Kurven. Leipziger Berichre, Math Phys Klasse. 68, 23-28 (1916)

6. Nilsrakoo, W, Saejung, S: The James constant of normalized norms on $R^{2}$. J Ineq Appl 2006, 1-12 (2006). Article ID 26265

7. Bonsall, FF, Duncan, J: Numerical ranges II. Lecture Note Series in London Mathematical Society. Cambridge University Press, Cambridge10 (1973)

8. Day, MM: Polygons circumscribed about closed convex curves. Trans Am Math Soc. 62, 315-319 (1947). doi:10.1090/ S0002-9947-1947-0022686-9

9. del Río, M: Ortogonalidad en Espacios Normados y Caracterización de Espacios Prehilbertianos. Dpto. de Análisis Matemático, Univ. de Santiago de Compostela, Spain, Serie B. 14 (1975)

doi:10.1186/1029-242X-2011-2

Cite this article as: Alonso: Any two-dimensional Normed space is a generalized Day-James space. Journal of Inequalities and Applications 2011 2011:2.

\section{Submit your manuscript to a SpringerOpen ${ }^{\circ}$} journal and benefit from:

- Convenient online submission

- Rigorous peer review

- Immediate publication on acceptance

- Open access: articles freely available online

- High visibility within the field

- Retaining the copyright to your article

Submit your next manuscript at $\boldsymbol{s p r i n g e r o p e n . c o m ~}$ 